\title{
Multispeed Lattice Boltzmann Model with Space-Filling Lattice for Transcritical Shallow Water Flows
}

\author{
Y. Peng, ${ }^{1}$ J. P. Meng, ${ }^{2}$ and J. M. Zhang ${ }^{1}$ \\ ${ }^{1}$ State Key Laboratory of Hydraulics and Mountain River Engineering, Sichuan University, Chengdu 610065, China \\ ${ }^{2}$ Scientific Computing Department, STFC Daresbury Laboratory, Warrington WA4 4AD, UK
}

Correspondence should be addressed to J. M. Zhang; zhangjianmin@scu.edu.cn

Received 21 February 2017; Revised 7 June 2017; Accepted 5 July 2017; Published 24 August 2017

Academic Editor: Ling Qian

Copyright (C) 2017 Y. Peng et al. This is an open access article distributed under the Creative Commons Attribution License, which permits unrestricted use, distribution, and reproduction in any medium, provided the original work is properly cited.

Inspired by the recent success of applying multispeed lattice Boltzmann models with a non-space-filling lattice for simulating transcritical shallow water flows, the capabilities of their space-filling counterpart are investigated in this work. Firstly, two lattice models with five integer discrete velocities are derived by using the method of matching hydrodynamics moments and then tested with two typical 1D problems including the dam-break flow over flat bed and the steady flow over bump. In simulations, the derived space-filling multispeed models, together with the stream-collision scheme, demonstrate better capability in simulating flows with finite Froude number. However, the performance is worse than the non-space-filling model solved by finite difference scheme. The stream-collision scheme with second-order accuracy may be the reason since a numerical scheme with second-order accuracy is prone to numerical oscillations at discontinuities, which is worthwhile for further study.

\section{Introduction}

The shallow water equations (SWEs) have been used to model free surface flows in rivers and coastal areas under the assumption of the hydrostatic pressure [1]. Numerically, the SWEs can be solved by using conventional numerical method such as finite difference methods [2], finite element methods [3], and finite volume methods [4]. Alternatively, the lattice Boltzmann method (LBM) [5-8] can be used to model SWEs at mesoscopic level [1,9-18]. In particular, one-dimensional shallow water flows have been studied by Frandsen [19], Thang et al. [20], and Chopard et al. [21].

A major limitation of LBM is its inability to model supercritical flows. For this reason, Chopard et al. [21] developed an asymmetric lattice Boltzmann model for one-dimensional flow flows which can simulate flows with Froude numbers larger than 1. La Rocca et al. [22] proposed a multispeed model with a non-space-filling lattice which is solved by finite difference scheme and successfully simulated supercritical flows. Here, by using the word "multispeed," it means lattices that have more than one nonzero speed in one dimension; see, for example, La Rocca et al. [22] and Brownlee et al. [23]. For instance, the commonly used D1Q3, D2Q9, and
D3Q19 lattices are not classified as multispeed lattice. Also, "non-space-filling"/“space-filling" means a lattice model that cannot/can fit into the standard steam-collision scheme.

Inspired by the success of the non-space-filling multispeed model, the capability of their space-filling counterpart will be investigated on simulating flows with finite Froude number. Specifically, two space-filling models will be derived by matching hydrodynamic moments (see, e.g., $[1,22,24]$ ) and then tested by using two typical shallow water problems.

\section{Multispeed Lattice Boltzmann Models for 1D Shallow Water Equation}

The one-dimensional SWEs read

$$
\begin{aligned}
\frac{\partial h}{\partial t}+\frac{\partial(h u)}{\partial x}= & 0, \\
\frac{\partial(h u)}{\partial t}+\frac{\partial\left(h u^{2}\right)}{\partial x}= & -g \frac{\partial}{\partial x}\left(\frac{h^{2}}{2}\right)+\nu \frac{\partial^{2}(h u)}{\partial x^{2}}-g h \frac{\partial z_{b}}{\partial x} \\
& +F
\end{aligned}
$$


which describe the evolution of water depth $h$ and depthaveraged velocity $u$. The force term $F$ is used to model various interesting effects such as wind-induced surface stress and the bed gradient.

SWEs can be modelled by using a mesoscopic evolution rule

$$
\begin{aligned}
f_{\alpha}\left(x+e_{\alpha} \Delta t, t+\Delta t\right)-f_{\alpha}(x, t) \\
=-\frac{1}{\tau}\left(f_{\alpha}-f_{\alpha}^{\mathrm{eq}}\right)+\Delta t S_{\alpha}\left(x+\frac{1}{2} e_{\alpha} \Delta t, t\right)
\end{aligned}
$$

which describes the fluid motion using a distribution function $f$. Important factors, such as wind-induced surface stress, are also modelled by a force term at the right hand side; that is, $S_{\alpha}=w_{\alpha} e_{a i} F_{i} /(\mathrm{cs})^{2}$ and $F_{i}=F_{i}\left(x+(1 / 2) e_{\alpha} \Delta t, t\right)$. The weight factor is denoted by $w_{\alpha}$ for a discrete velocity $e_{\alpha}$, and the sound speed cs can be calculated by using $e / \lambda$, where $\lambda$ is a reference quantity varying with lattice.

To successfully simulate shallow water flows, the key is to define an appropriate local equilibrium function $f_{\alpha}^{\mathrm{eq}}$ and the associated lattice. Here, the equilibrium distribution function in Zhou [1] is generalized to

$$
\begin{aligned}
& f_{\alpha}^{\mathrm{eq}} \\
& = \begin{cases}w_{0} h\left(\frac{1}{w_{0}}-A \frac{g h}{e^{2}}-\frac{u_{i} u_{i}}{2 \mathrm{cs}^{2}}\right) & \alpha=0, \\
w_{\alpha} h\left(B \frac{g h}{e^{2}}+\frac{e_{\alpha i} u_{i}}{\mathrm{cs}^{2}}+\frac{1}{2}\left[\frac{e_{\alpha i} e_{\alpha j} u_{i} u_{j}}{\mathrm{cs}^{4}}-\frac{u_{i} u_{i}}{\mathrm{cs}^{2}}\right]\right) & \alpha \neq 0,\end{cases}
\end{aligned}
$$

where the coefficients $A$ and $B$ can be adjusted to satisfy the conservation of mass and momentum, i.e.,

$$
\begin{aligned}
h & =\sum_{\alpha} f_{\alpha}^{\mathrm{eq}}, \\
h u^{2}+\frac{g h^{2}}{2} & =\sum_{\alpha} e_{\alpha} e_{\alpha} f_{\alpha}^{\mathrm{eq}} .
\end{aligned}
$$

By substituting (3) into (4), the following equations can be obtained:

$$
\begin{aligned}
w_{0} A & =\sum_{\alpha} w_{\alpha} B \quad(\alpha \neq 0), \\
\sum_{\alpha} \frac{e_{\alpha} e_{\alpha} w_{\alpha} B}{e^{2}} & =\frac{1}{2} \quad(\alpha \neq 0) .
\end{aligned}
$$

The weights $w_{\alpha}$ in (3) will depend on the choice of lattice. Here two sets of velocity lattices will be adopted, that is, discrete velocities $(0, \pm 1, \pm 2)$ derived by Qian and Zhou [25] and $(0, \pm 1, \pm 3)$ derived by Chikatamarla and Karlin [26]. They are named as D1Q5A and D1Q5B, respectively, and the relevant parameters are listed in Table 1 . For convenience, the standard $(0, \pm 1)(\mathrm{D} 1 \mathrm{Q} 3)$ lattice also is listed and it will be used for comparison in the following simulations.

\section{Case 1: 1D Dam-Break Flow}

To test the ability of the derived space-filling models, firstly a simple but challenging $1 \mathrm{D}$ dam-break flow over flat bed
TABLE 1: Parameters of three lattice Boltzmann models. The reference quantity and the weights for D1Q5A and D1Q5B are adopted from Qian and Zhou [25] and Chikatamarla and Karlin [26], respectively.

\begin{tabular}{lccc}
\hline & D1Q3 & D1Q5A & D1Q5B \\
\hline$A$ & $3 / 4$ & $1 / 2$ & $(110+23 \sqrt{10}) / 48$ \\
$B$ & $3 / 2$ & $1 / 2$ & $(5-\sqrt{10}) / 6$ \\
$\lambda$ & $\sqrt{3}$ & 1 & $\sqrt{(5-\sqrt{10}) / 3}$ \\
$w(0)$ & $2 / 3$ & $1 / 2$ & $(16-4 \sqrt{10}) / 45$ \\
$w( \pm 1)$ & $1 / 6$ & $1 / 6$ & $(24+3 \sqrt{10}) / 80$ \\
$w( \pm 2)$ or $w( \pm 3)$ & - & $1 / 12$ & $(16+5 \sqrt{10}) / 720$ \\
\hline
\end{tabular}

is used. Initially, the water is stationary but there is a discontinuity in the middle of computational region $(x=$ $1000 \mathrm{~m}$ ), described as follows:

$$
h(x)= \begin{cases}h_{1}, & 0 \leq x \leq 1000 \mathrm{~m} \\ h_{2}, & 1000 \mathrm{~m}<x \leq 2000 \mathrm{~m}\end{cases}
$$

The discontinuity will then propagate stream-wise and change its shape. The sharp gradient at the wave front is difficult to be captured smoothly.

In simulations, 2000 cells are used and therefore $\Delta x$ is $1 \mathrm{~m}$ and $\Delta t$ is $0.1 \mathrm{~s}$. The initial upstream water depth is set to be $5 \mathrm{~m}$ for all tests. Firstly, in order to verify the two multispeed lattice models, the water depth of $3 \mathrm{~m}$ is specified in the downstream, where the maximum Froude (Fr) number is 0.26 . In Figure 1, the predictions of water depth and velocity of two models and the D1Q3 model are compared with the corresponding analytical solutions [27]. It is shown that all three models are satisfactory, which is as expected since the Fr is relatively low.

To examine the capability of simulating flow with higher Froude number, a series of tests will be carried out by adjusting the downstream depth. It is found, as shown in Figure 2, that the D1Q5B model can simulate flows with Fr $=1.14$ if the relaxation time is set to be 1.99 , although there are errors near the discontinuity perhaps due to the numerical oscillations. The D1Q5A is able to simulate flows with Fr $=1.07$, while the D1Q3 model fails to simulate Froude number beyond unity. The difference between two multispeed models shows that the capability can also be impacted by the lattice structure.

\section{Case 2: 1D Steady Flow over Bump}

A steady flow over a bump bed will be utilized to further test the multispeed models. For this flow problem, the subcritical flow over the same bump has been studied by Goutal and Maurel [28], Zhou [1], and Peng et al. [18].

For testing, the bed bottom is defined as

$$
Z_{b}(x)= \begin{cases}0.2-0.05(x-6)^{2}, & 8 \mathrm{~m}<x<12 \mathrm{~m} \\ 0, & \text { else. }\end{cases}
$$

The inlet boundary condition is specified by setting a constant discharge of $Q=4.42 \mathrm{~m}^{2} / \mathrm{s}$. The length of channel is $25 \mathrm{~m}$. 


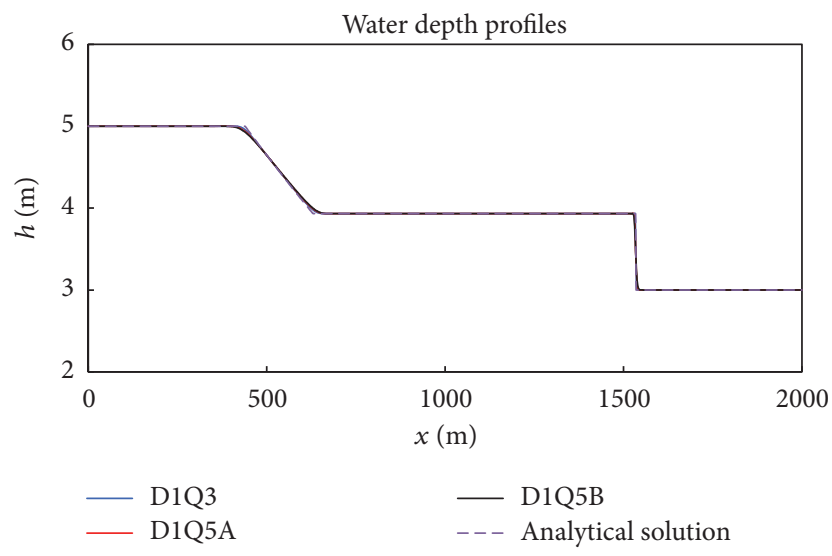

(a)

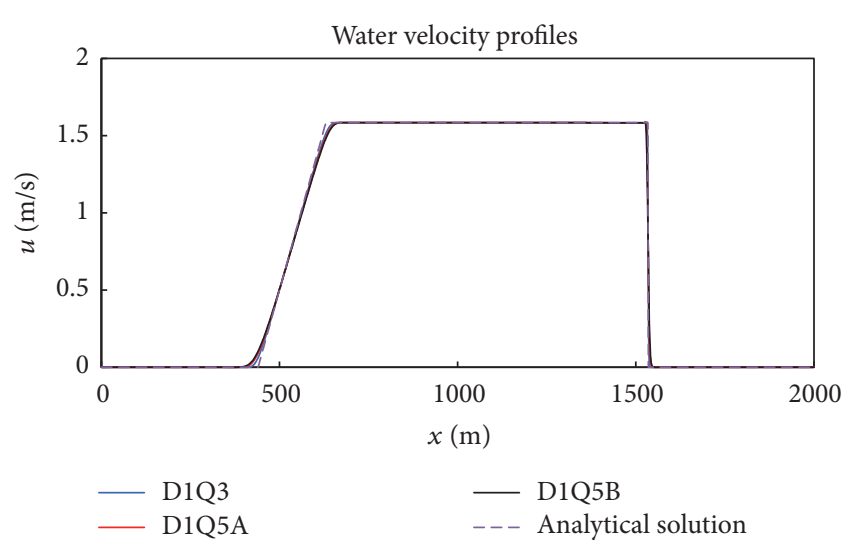

(b)

FIGURE 1: Water depth and velocity profiles predicted by D1Q3, D1Q5A, and D1Q5B models at $t=80 \mathrm{~s}\left(h_{1}=5 \mathrm{~m}\right.$ and $\left.h_{2}=3\right)$.

A fixed water depth $h_{0}$ will be used at the outlet boundary, which will be reduced gradually to find the maximum Fr that can be simulated. The space step $\Delta x$ is set to be $0.1 \mathrm{~m}$ to get the grid-independent solution according to Peng et al. [18] and the time step $\Delta t=0.002 \mathrm{~s}$. A relaxation time of 1.99 is employed for all simulations except for the cases shown in Figure 3. Besides, the bed friction is ignored here.

First, the case with a downstream water depth of $2 \mathrm{~m}$ is tested to verify the multispeed models. As shown in Figure 3, all models agree well with the analytical solution, which again confirms capability of multispeed model in the relative low Froude number.

Then the downstream water depth is adjusted to test the capability of higher Froude number, that is, to find the minimal downstream water depth. The corresponding results are presented in Figure 4. It is found that the maximum Fr for D1Q3 is 0.69 , which is consistent with the previous findings by Zhou [1]. The D1Q5B model can simulate flows with a Froude number of 1.09, while the D1Q5A model fails to simulate Froude number beyond 0.91 , which also shows the importance of lattice structure.

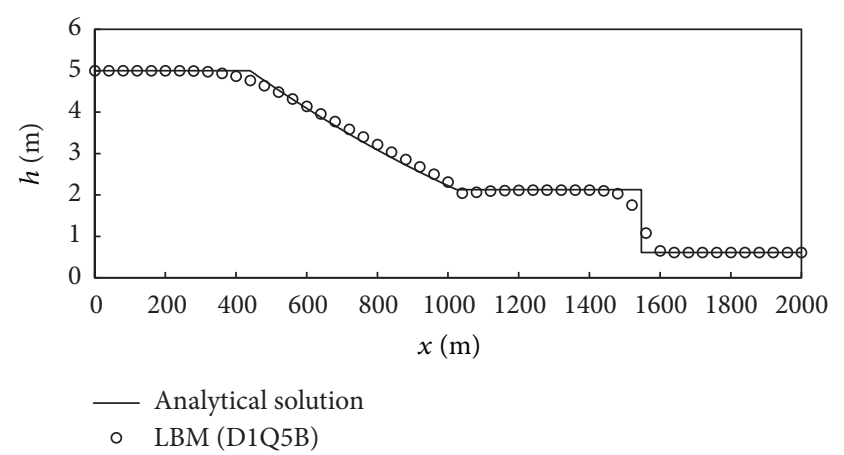

(a)

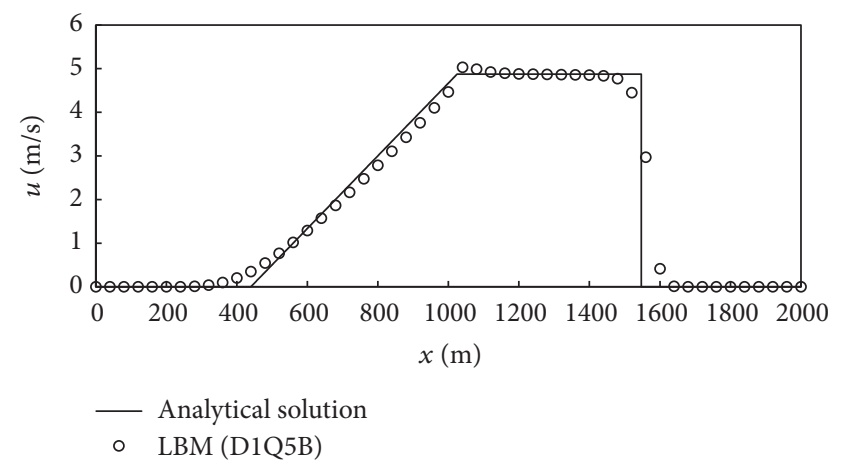

(b)

FIGURE 2: Water depth and velocity profiles predicted by D1Q5B models at $t=80 \mathrm{~s}$ with $h_{2}=0.61 \mathrm{~m}$ and maximum $\mathrm{Fr}=1.14$ [(a) water depth, (b) water velocity].

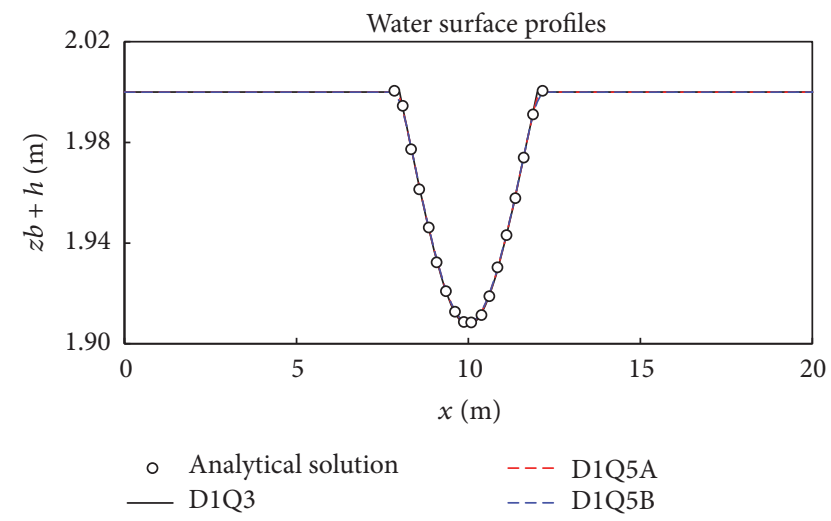

FIgURE 3: Water surface profiles with $h_{0}=2 \mathrm{~m}$.

\section{Concluding Remarks}

In this study, the space-filling multispeed lattice Boltzmann model has been investigated to simulate the transcritical shallow water flows. By matching hydrodynamics moments, two lattice models with five integer lattice velocities have been derived, which are tested together with the standard model with three discrete velocities for a $1 \mathrm{D}$ dam-break and a $1 \mathrm{D}$ steady flow over bump.

It is encouraging to find that the space-filling multispeed model can improve the capability of simulating flows with 


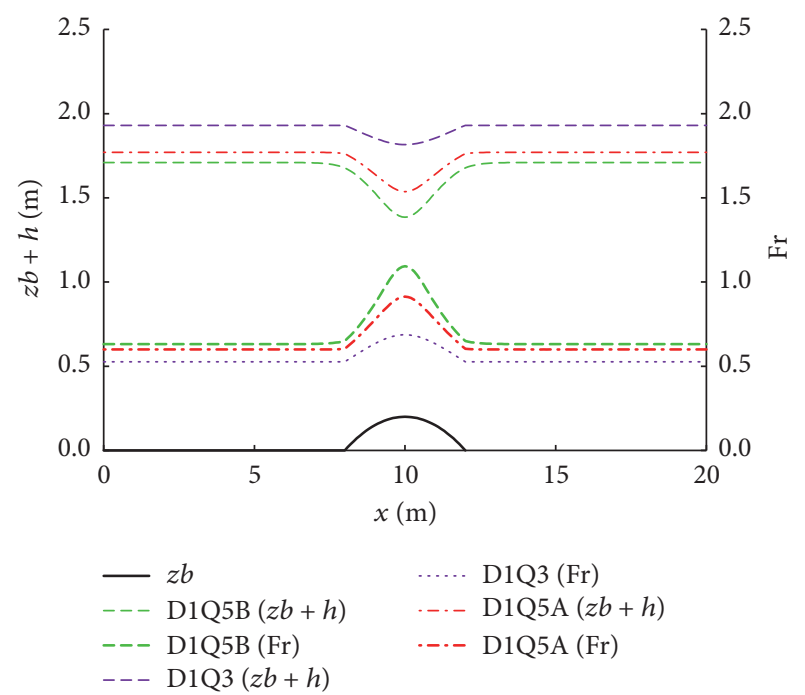

FIGURE 4: Water surface and Froude number profiles with minimum water depth for three models $\left(h_{0}=1.93,1.77\right.$, and 1.71 and $\mathrm{Fr}=0.69$, 0.91, and 1.09 for D1Q3, D1Q5A, and D1Q5B models, resp.).

higher Fr, particularly compared with the standard D1Q3 model. The different performances between two multispeed models show that capability can be impacted by the lattice structure.

In comparison with the non-space-filling model proposed by La Rocca et al. [22], the space-filling versions here demonstrate limited capability in terms of finite Froude number although similar numbers of discrete velocities are used in these two studies. This is potentially due to the fact that the stream-collision scheme is a second-order scheme. It is well known that a numerical scheme of second-order accuracy is prone to numerical oscillations at discontinuities. The impact of the stream-collision scheme will be studied further in the future.

\section{Conflicts of Interest}

The authors declare that they have no conflicts of interest.

\section{Acknowledgments}

Y. Peng would like to acknowledge the financial support of National Natural Science Foundation of China (Grant nos. 51409183, 51579166, and 51611130203). J. P. Meng is grateful to the support of the Open Fund from the State Key Laboratory of Hydraulics and Mountain River Engineering in Sichuan University, China (Grant no. SKHL1410). Both Y. Peng and J. P. Meng would like to acknowledge the support of International Exchange Programme of Royal Society, UK (IE151119) and NSFC, China (51611130203).

\section{References}

[1] J. G. Zhou, Lattice Boltzmann Methods for Shallow Water Flows, Springer, Berlin, Germany, 2004.
[2] V. Casulli and R. T. Cheng, "Semi-implicit finite difference methods for three-dimensional shallow water flow," International Journal for Numerical Methods in Fluids, vol. 15, no. 6, pp. 629-648, 1992.

[3] T. W. H. Sheu and C. C. Fang, "High resolution finite-element analysis of shallow water equations in two dimensions," Computer Methods in Applied Mechanics and Engineering, vol. 190, no. 20-21, pp. 2581-2601, 2001.

[4] A. H. Daoud, K. A. Rakha, and A. G. Abul-azm, "A twodimensional finite volume hydrodynamic model for coastal areas: model development and validation," Ocean Engineering, vol. 35, no. 1, pp. 150-164, 2008.

[5] F. J. Higuera and S. Succi, "Simulating the flow around a circular cylinder with a lattice boltzmann equation," Europhysics letters, vol. 8, no. 6, pp. 517-521, 1989.

[6] R. Benzi, S. Succi, and M. Vergassola, "The lattice Boltzmann equation: theory and applications," Physics Reports, vol. 222, no. 3, pp. 145-197, 1992.

[7] S. Chen and G. D. Doolen, "Lattice Boltzmann method for fluid flows," Annual Review of Fluid Mechanics, vol. 30, pp. 329-364, 1998.

[8] C. K. Aidun and J. R. Clausen, "Lattice-boltzmann method for complex flows," Annual Review of Fluid Mechanics, vol. 42, pp. 439-472, 2010.

[9] R. Salmon, "The lattice Boltzmann method as a basis for ocean circulation modeling," Journal of Marine Research, vol. 57, no. 3, pp. 503-535, 1999.

[10] P. J. Dellar, "Nonhydrodynamic modes and a priori construction of shallow water lattice Boltzmann equations," Physical Review E-Statistical, Nonlinear, and Soft Matter Physics, vol. 65, no. 3, Article ID 036309, pp. 1-12, 2002.

[11] H. Liu, J. G. Zhou, and R. Burrows, "Numerical modeling of turbulent compound channel flow using the lattice Boltzmann method," International Journal for Numerical Methods in Fluids, vol. 59, no. 7, pp. 753-765, 2009.

[12] H. Liu, J. G. Zhou, and R. Burrows, "Multi-block lattice Boltzmann simulations of subcritical flow in open channel junctions," Computers and Fluids, vol. 38, no. 6, pp. 1108-1117, 2009.

[13] C. Biscarini, S. D. Francesco, and M. Mencattini, "Application of the lattice Boltzmann method for large-scale hydraulic problems," International Journal of Numerical Methods for Heat and Fluid Flow, vol. 21, no. 5, pp. 584-601, 2011.

[14] K. R. Tubbs and F. T. Tsai, "GPU accelerated lattice Boltzmann model for shallow water flow and mass transport," International Journal for Numerical Methods in Engineering, vol. 86, no. 3, pp. 316-334, 2011.

[15] C. Biscarini, S. D. Francesco, F. Nardi, and P. Manciola, "Detailed simulation of complex hydraulic problems with macroscopic and mesoscopic mathematical methods," Mathematical Problems in Engineering, vol. 2013, Article ID 928309, 14 pages, 2013.

[16] Y. Peng, J. G. Zhou, and R. Burrows, "Modelling solute transport in shallow water with the lattice Boltzmann method," Computers and Fluids, vol. 50, pp. 181-188, 2011.

[17] Y. Peng, J. G. Zhou, J. M. Zhang, and H. Liu, "Lattice Boltzmann modeling of shallow water flows over discontinuous beds," International Journal for Numerical Methods in Fluids, vol. 75, no. 8, pp. 608-619, 2014.

[18] Y. Peng, J. M. Zhang, and J. G. Zhou, "Lattice boltzmann model using two relaxation times for shallow-water equations," Journal 
of Hydraulic Engineering, vol. 142, no. 2, Article ID 06015017, 2015.

[19] J. B. Frandsen, "A simple LBE wave runup model," Progress in Computational Fluid Dynamics, vol. 8, no. 1-4, pp. 222-232, 2008.

[20] P. V. Thang, B. Chopard, L. Lefévre, D. A. Ondo, and E. Mendes, "Study of the 1D lattice Boltzmann shallow water equation and its coupling to build a canal network," Journal of Computational Physics, vol. 229, no. 19, pp. 7373-7400, 2010.

[21] B. Chopard, P. V. Thang, and L. Lefèvre, "Asymmetric lattice Boltzmann model for shallow water flows," Computers and Fluids, vol. 88, pp. 225-231, 2013.

[22] M. La Rocca, A. Montessori, P. Prestininzi, and S. Succi, "A multispeed Discrete Boltzmann Model for transcritical 2D Shallow water flows," Journal of Computational Physics, vol. 284, pp. 117-132, 2015.

[23] R. A. Brownlee, A. N. Gorban, and J. Levesley, "Stable multispeed lattice Boltzmann methods," Tech. Rep. MA-06-026, University of Leicester, Leicester, UK, 2006, https://arxiv.org/abs/ cond-mat/0611616.

[24] H. W. Zheng, C. Shu, Y. T. Chew, and J. Qiu, "A platform for developing new lattice Boltzmann models," International Journal of Modern Physics C. Computational Physics and Physical Computation, vol. 16, no. 1, pp. 61-84, 2005.

[25] Y.-H. Qian and Y. Zhou, "Complete Galilean-invariant lattice BGK models for the Navier-Stokes equation," Europhysics Letters, vol. 42, no. 4, pp. 359-364, 1998.

[26] S. S. Chikatamarla and I. V. Karlin, "Entropy and Galilean invariance of lattice Boltzmann theories," Physical Review Letters, vol. 97, no. 19, Article ID 190601, 2006.

[27] O. Delestre, C. Lucas, P.-A. Ksinant et al., "SWASHES: a compilation of shallow water analytic solutions for hydraulic and environmental studies," International Journal for Numerical Methods in Fluids, vol. 72, no. 3, pp. 269-300, 2013.

[28] N. Goutal and F. Maurel, Proceedings of the 2nd Workshop on Dam-break Wave Simulation, Tech. Rep. HE-43/97/016/B, Département Laboratoire National d'Hydraulique, Groupe Hydraulique Fluviale, Electricité de France, France, 1997. 


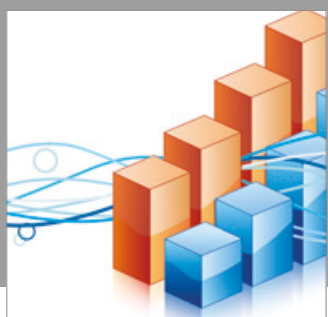

Advances in

Operations Research

vatersals

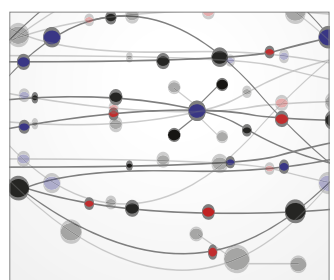

\section{The Scientific} World Journal
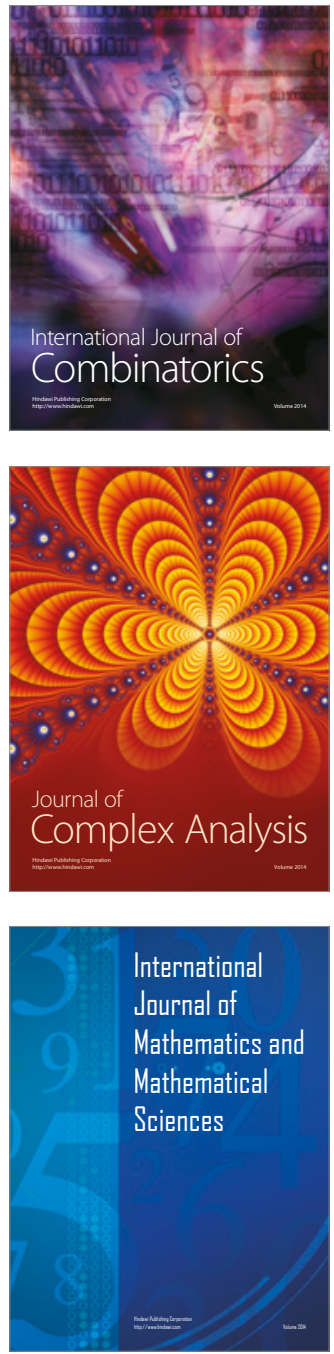
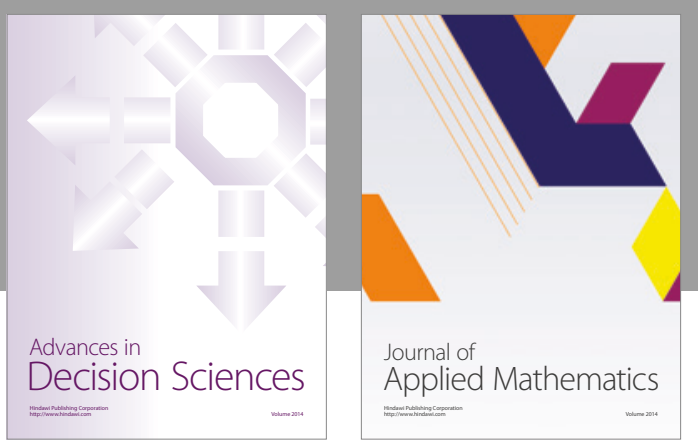

Algebra

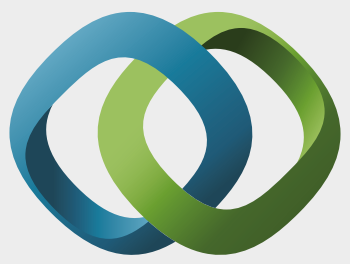

\section{Hindawi}

Submit your manuscripts at

https://www.hindawi.com
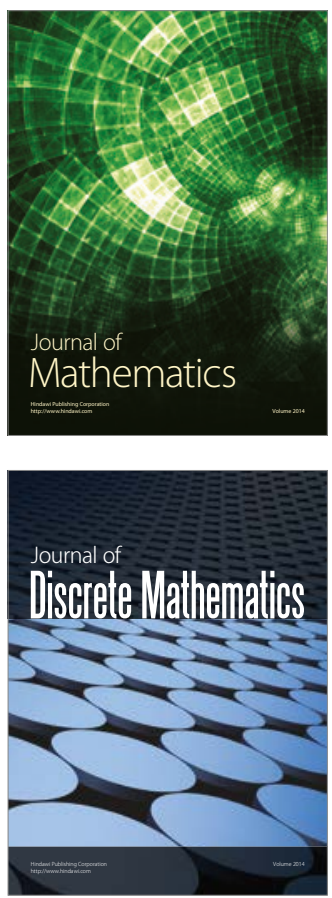

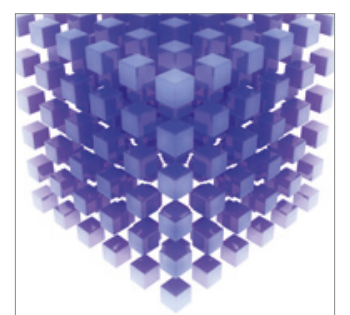

Mathematical Problems in Engineering
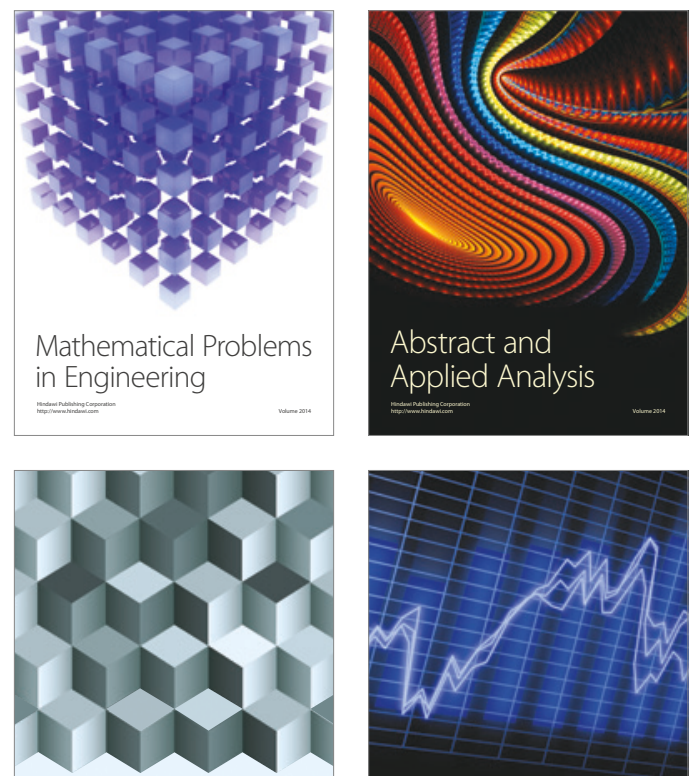

Journal of

Function Spaces

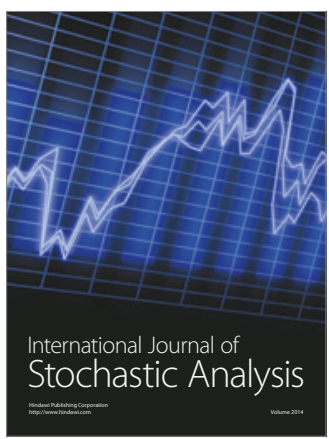

Probability and Statistics
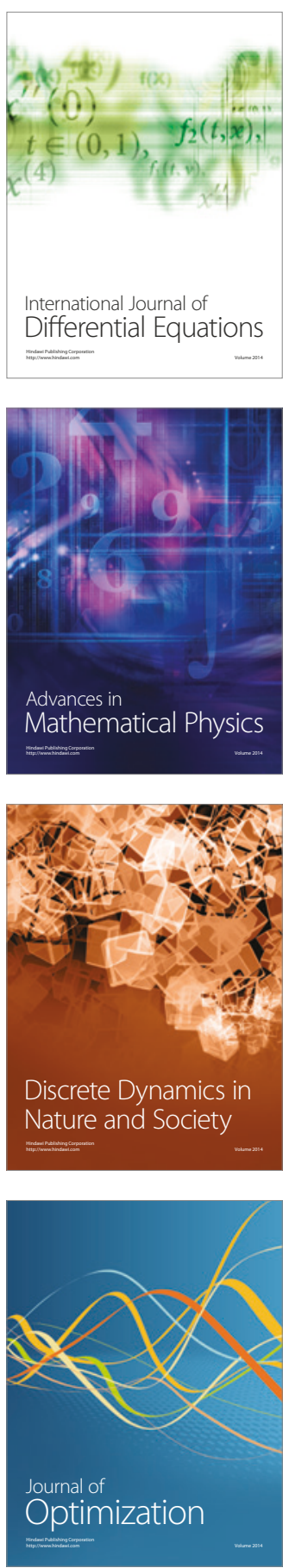